\title{
Les MRC rurales-urbaines et le Comité Ruest-Jutras et Nicolet
}

\author{
Jacques Léveillée \\ Université du Québec à Montréal
}

Il est probable que le gouvernement adoptera une bonne partie des recommandations formulées par le groupe de travail sur le fonctionnement des 28 MRC mixtes (rurales-urbaines). Ce «succès» de la commission Ruest-Jutras/Nicolet tient au fait que son mandat était bien défini et que les autorités gouvernementales étaient disposées à recevoir ses recommandations. Cette réussite procède également d'une écoute attentive et ouverte aux attentes et aux suggestions apportées par les élus rencontrés par le groupe de travail. Enfin, il importe de noter que la volonté de continuer à vivre ensemble était présente dans une majorité des MRC soumises à l'étude, malgré les tensions au sein de ces MRC, en particulier celles qui proviennent de l'insécurité, réelle ou perçue, des populations «dites rurales » de ces MRC.

Il arrive fréquemment que nous nous désespérions du fait que les élus attendent que les feux soient bien pris avant de faire appel aux pompiers. Ainsi en est-il dans le domaine des affaires municipales. Les responsables politiques ont tendance à attendre qu'un scandale éclate ou qu'une situation s'envenime avant de donner un mandat urgent à des fonctionnaires ou à d'autres types de consultants pour qu'ils élaborent, souvent en catastrophe, une solution au problème posé. Aussi, lorsque la réaction politique vient à l'encontre de cette pratique, il est intéressant d'en faire l'analyse et d'en tirer quelques conclusions quant aux impacts de cette façon, en théorie plus normale, de produire et de mettre en œuvre des politiques publiques.

\section{Le cas qui servira d'illustration s'inscrit dans la politique plus générale qui vise, depuis 1996, à consolider les entités municipales de base et les structures métropolitaines ou régionales dont elles font partie.}

Le cas qui servira d'illustration s'inscrit dans la politique plus générale qui vise, depuis 1996, à consolider les entités municipales de base et les structures métropolitaines ou régionales dont elles font partie. Il s'agit plus précisément de 28 de ces dernières structures qui ont comme principale caractéristique de réunir des municipalités représentant des populations «dites rurales» avec des municipalités administrant des services à l'intention de populations «dites urbaines». Comment ces municipalités risquent-elles de coexister si les réorganisations en cours se réalisent effectivement ? Est-il possible de concevoir et de mettre en oeuvre des aménagements techniques et politiques susceptibles de minimiser les heurts prévisibles?

Voilà deux des questions qui ont préoccupé les responsables administratifs et politiques du dossier de la réforme municipale au Québec avant de former un groupe de travail sur les MRC à caractère urbain et rural en avril 2001 dont le mandat central était de recommander des mesures afin de contrôler les brasiers avant que les feux n'éclatent. Ce groupe de travail, coprésidé par madame Francine Ruest-Jutras, mairesse de la Ville de Drummondville et monsieur Roger Nicolet, maire de Municipalité de Austin, a effectivement entrepris son travail à la mi-juillet 2001 et a remis son rapport à la ministre des Affaires municipales et de la Métropole à la mi-octobre 2001.

Après avoir présenté brièvement les MRC soumises à l'étude, le présent compte rendu de l'expérience du groupe de travail Ruest-Jutras/Nicolet fera état des principaux enjeux de coexistence que ce dernier a abordés, ainsi que les solutions qu'il a proposées en vue de les baliser ou de les solutionner. En conclusion, il sera question du sort qui fut réservé au rapport du groupe de travail, ce qui permettra une courte réflexion sur l'utilité d'une approche «prévisionnelle» eu égard à la formulation des politiques publiques. 


\section{Les 28 MRC soumises à l'attention du groupe de travail}

Parallèlement à une stratégie d'intervention sur le terrain municipal occupé par des municipalités rurales et par des MRC à caractère rural, le ministère des Affaires municipales et de la Métropole avait résolu de porter une attention toute particulière aux agglomérations urbaines, soit celles que l'on identifie comme les régions métropolitaines de recensement et comme les agglomérations de recensement. Ce sont ces dernières qui nous intéressent.

Les 28 MRC qui furent objets d'analyse et de recommandations de la part du groupe de travail Ruest-Jutras/Nicolet étaient donc composées, comme le veut la catégorie statistique à laquelle elles appartiennent, d'une ville de 10000 personnes et de municipalités adjacentes formant avec elle un ensemble soudé par des liens de mobilité, en particulier pour fin d'emploi. À la périphérie de cette agglomération de recensement, nous retrouvons les municipalités rurales qui ont historiquement été associées aux municipalités du noyau urbain au sein des 28 MRC qui sont, pour cette raison, des MRC mixtes.

Dans 12 des 28 MRC, les municipalités rurales concentrent plus de $50 \%$ de la population totale de leur MRC respective. Or, pour atteindre ce pourcentage, il faut évidemment qu'elles s'y mettent à plusieurs, soit toutes les municipalités de la MRC sauf la ville centre. En contrepartie, dans 16 des $28 \mathrm{MRC}$, la ville centre comprend, à elle seule, plus de $50 \%$ de la population totale de la MRC. Dans la majorité des situations, les municipalités rurales se retrouvent donc en présence d'un «partenaire» imposant, avant même que la fusion envisagée dans la stratégie gouvernementale ne soit réalisée. Dans quelques autres cas, soit ceux que l'on retrouve dans les MRC d'Autray, du Centre-de-la-Mauricie, de Joliette, de Beauharnois-Salaberry, de Rivière-du-Nord et de BromeMissisquoi, on évite le «partenaire» urbain unique, puisqu'il existe au moins deux villes de taille et de rôle comparables, ce qui laisse présager une dynamique de coexistence différente.

Ce déséquilibre démographique entre ruraux et urbains ne peut que s'amplifier, en faveur des « urbains », à l'issue du regroupement des municipalités formant l'agglomération urbaine.
Ce déséquilibre démographique entre ruraux et urbains ne peut que s'amplifier, en faveur des «urbains », à l'issue du regroupement des municipalités formant l'agglomération urbaine. Or, ce déséquilibre actuel ou appréhendé ne vient pas seul. Il est couplé à un déséquilibre fiscal ou financier qui, en règle générale, défavorise les municipalités périphériques des MRC. Enfin, ces deux déséquilibres sont accompagnés de leur corollaire politique et s'expriment dans les rapports de force lors des prises de décision au sein des MRC. Ce dernier point est au cœur de la problématique étudiée par le groupe de travail Ruest-Jutras/Nicolet.

Certes, avant le processus de regroupement des agglomérations de recensement pour consolider les villes centres, aucune des villes centres des 28 MRC ne contrôlait systématiquement le processus de décision en vigueur dans les MRC. Celui-ci obéit à la formule de la double majorité. Pour qu'une décision soit adoptée, il faut qu'elle obtienne la double majorité des votes, soit celle qui répond au poids démographique, d'une part, et celle qui prend sa source dans les lettres patentes et qui attribue un poids politique à chacun des partenaires, d'autre part. Ainsi, sauf une exception, les lettres patentes n'accordaient la majorité politique à aucune ville centre. Donc, pas de possibilité de veto de ce côté. Par contre, la majorité démographique ne pouvait pas ne pas faire sentir toute sa lourdeur. Elle donnait, de facto, un pouvoir de veto aux villes centres représentant plus de la moitié de la population de la MRC. Or, cet état de fait était appelé à se généraliser suite aux regroupements des agglomérations de recensement.

Possibilité de veto ne veut pas dire utilisation fréquente ou systématique du veto. Déséquilibres démographique, financier et politique ne conduisent pas inévitablement ou perpétuellement à des situations de domination et de mauvaises fréquentations. Par contre, ils peuvent générer des déséquilibres tenaces, persistants, têtus entre les faits et les perceptions de ces derniers. Le milieu municipal ne détient probablement pas le record de ces derniers déséquilibres. Mais il n'est pas non plus en reste sur ce plan. Aussi, le groupe de travail n'a pas eu à traiter que de situations conflictuelles ou minées par suite des affrontements «structurels » et «culturels » entre les partenaires ruraux et urbains au sein des 28 MRC à l'étude. Par ailleurs, les perceptions des uns à l'égard des autres, et surtout les perceptions au sujet de ce qui était en train de se tramer au plan de la réforme municipale, coloraient et colorent toujours les attitudes quant à la 
volonté et quant à la capacité de vivre ensemble au sein des MRC. La crainte d'être manipulés par les «urbains » et par les réformateurs gouvernementaux est très présente chez les «ruraux ». La crainte d'être empêchés d'avancer par les «ruraux» est aussi fort présente chez les «urbains».

\section{La crainte d'être manipulés par les « urbains » et par les réformateurs gouvernementaux est très présente chez les « ruraux». La crainte d'être empêchés d'avancer par les « ruraux» est aussi fort présente chez les « urbains ».}

Le mandat principal du groupe de travail n'était pas de traiter spécifiquement de la problématique des «ruraux » au sein des 28 MRC. Il l'a tout de même fait parce qu'une bonne partie des difficultés de fonctionnement des 28 MRC provient du fait que les « ruraux » hésitent à investir ces structures décisionnelles pour les raisons que nous évoquerons dans un instant. Au terme de cette incursion, nous aborderons plus directement les mandats confiés au groupe de travail.

\section{Les orphelines de la réforme municipale}

Amorcée au milieu des années 1960, la réorganisation municipale a d'abord mis le cap du côté des municipalités rurales. La modernisation de la société québécoise semblait exiger, comme c'était le cas dans des sociétés comparables, une adaptation des administrations publiques aux nouvelles réalités et aux nouveaux défis. L'objectif était alors de favoriser une diminution du nombre d'intervenants dans l'espoir que ces derniers soient mieux outillés pour contribuer à la modernisation de leurs sociétés locales, tout en contribuant au mouvement plus général.

Cette stratégie n'a pas donné les résultats escomptés. Aussi, sans délaisser tout à fait leurs intentions à cet égard, et sans qu'il y ait de relation de cause à effet entre les deux stratégies, les réformateurs gouvernementaux se tournèrent progressivement du côté des milieux urbains.

La prise de conscience du fait urbain québécois fut relativement lente à se manifester. Il aura fallu au moins une bonne dizaine de commissions ou groupes d'étude, de la Commission Tremblay au milieu des années 1950 à la Commission Parizeau au milieu des années 1980, pour que le gouvernement québécois s'engage résolument à stimuler et à rajeunir l'ensemble des agglomérations urbaines du Québec. Aidé en cela par les revendications de la trentaine de villes centres du Québec, le gouvernement prit le virage urbain et mit l'adaptation des structures administratives urbaines au premier rang de son agenda politique et administratif. De la «réforme Ryan» à la réforme Harel, en passant par les trois volets du ministre Trudel, un fil conducteur lie le tout. Pour répondre aux défis de la concurrence économique, de la croissance urbaine, de la gestion efficace et efficiente des services publics urbains actuels et de ceux qu'il faudra développer pour circonscrire et solutionner les problèmes sociaux que les milieux urbains concentrent, il faut repenser et restructurer les agglomérations urbaines.

Dans le discours et dans la mise en œuvre de cette politique urbaine, la restructuration passe invariablement par le regroupement des unités municipales formant agglomérations. Mais elle passe également par des invitations de plus en plus pressantes adressées aux partenaires des MRC pour qu'ils trouvent des façons de mettre en commun des services, des équipements supralocaux, des expertises, des ressources financières, etc. Ce sont effectivement ces invitations, et la croyance qu'elles sont faites de connivence entre réformateurs gouvernementaux et leaders urbains, qui alimentent les craintes, les hésitations et les perceptions plus ou moins fondées des «ruraux » à l'égard de tout ce qui s'élabore et se met en marche en matière de réforme municipale.

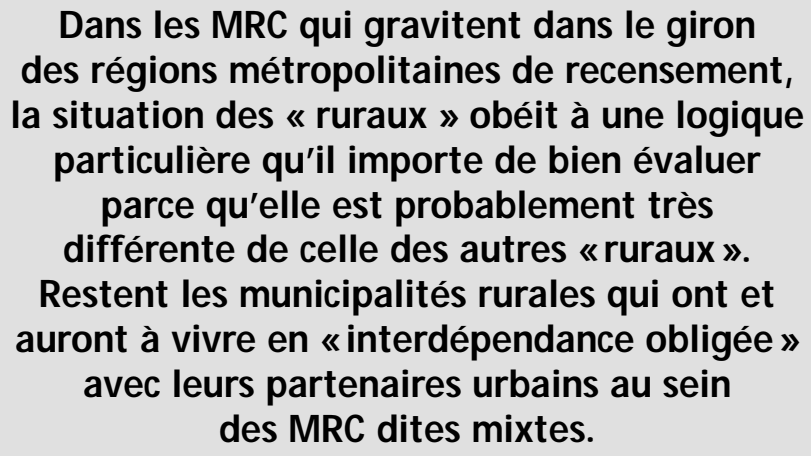

Dans les MRC exclusivement rurales, cette tension n'existe pas. De plus, la Loi 29 et l'énoncé de politique sur la ruralité contribueront probablement à faire en sorte que les municipalités rurales et leurs instances régionales, dont les MRC, se prennent en charge et conçoivent des solutions en réponse à leurs besoins et à leur capacité de payer. Dans les MRC qui gravitent dans le giron des régions métropolitaines de recensement, la situation des « ruraux » obéit à une logique particulière qu'il importe de bien évaluer parce qu'elle est probablement très diffé- 
rente de celle des autres « ruraux ». Restent les municipalités rurales qui ont et auront à vivre en «interdépendance obligée » avec leurs partenaires urbains au sein des MRC dites mixtes.

Ces municipalités seront inévitablement moins nombreuses et représenteront moins de personnes. En faisant l'hypothèse que toutes les agglomérations de recensement des 28 MRC soient regroupées, le groupe de travail procédait à l'évaluation suivante :

«Les populations des municipalités rurales diminueraient de près de $40 \%$, passant ainsi de 729400 à 446305 personnes. Ceci entraînerait, par conséquent, une diminution du nombre de municipalités locales qui passerait de 361 à 298 , soit une diminution de $18 \%$. Nous estimons que, dans $46 \%$ des MRC étudiées, les municipalités rurales compteraient désormais pour moins de $30 \%$ de la population totale de la MRC. Dans cinq de ces MRC, la population représentée par des municipalités rurales n'atteindrait pas $20 \%$ de la population totale de la MRC. » (p. 10)

Le sentiment d'être étrangères aux préoccupations et aux aspirations des partenaires urbains de la MRC ne risque-t-il pas d'être encore plus prononcé chez les municipalités rurales restantes? Tout comme des orphelines, abandonnées dans le champ, par leurs pères et mères administratifs? L'expression de tels sentiments ne risquet-elle pas, en retour, d'impatienter les leaders des municipalités urbaines à l'endroit des leaders ruraux ? Est-ce que le biais industriel et urbain attribué aux MRC mixtes par les ruraux sera encore amplifié, laissant encore plus dans l'ombre les préoccupations agricoles et rurales de ces MRC? Autant de questions qui ont incité le groupe de travail à aborder les questions plus techniques de son mandat dans une perspective politique ouverte.

\section{M ariage de raison ou divorce à l'amiable?}

La coexistence des ruraux et des urbains au sein des 28 MRC dites mixtes a convaincu certains qu'il valait mieux tenter sa chance avec une stratégie différente de celle qui avait conduit au mariage de raison, par les bons soins du pouvoir gouvernemental au tournant des années 1980.

Une de ces stratégies a consisté, pour deux MRC, soit les MRC d'Autray et de Bécancour, de demander au groupe de travail de plaider en leur faveur, auprès de la ministre, un changement de statut et un rattachement au groupe des 50 MRC rurales. Pour trois autres MRC, le désir exprimé était également de procéder à un changement de statut, cette fois pour abolir la MRC actuelle au profit d'une MRC, une ville. Il s'agit des MRC de Rouyn-Noranda, du Centre-de-la-Mauricie et du HautSaint-Maurice. Dans ces deux derniers cas, les quelques municipalités rurales qui ne seraient pas partie à la nouvelle entente se rattacheraient à des MRC rurales voisines. Selon une même logique, il n'est pas interdit de penser que certaines municipalités rurales estimeront plus pertinent de se fondre à l'agglomération de recensement en voie de se transformer en une seule ville plutôt que de tenter un rattachement à une MRC rurale.

Une deuxième stratégie, en apparence moins radicale, procède sensiblement de la même prise de conscience quant à la difficulté de perpétuer, sous sa forme actuelle, le mariage de raison intervenu il y a trente ans. Plutôt que de se laisser convaincre par le gouvernement ou par d'autres intervenants qu'il faut mettre de plus en plus de services et équipements en commun, les tenants de cette stratégie, connue sous le nom de «formule Arthabaska» ou «formule de Victoriaville» et promue par un regroupement de villes centres, pourquoi ne pas arrêter toute cette agitation et repartir à neuf? Un nouveau départ qui se ferait en redonnant à chacun des deux secteurs en présence son autonomie de fonctionnement et en instituant, pour un nombre limité de domaines d'intervention, dont l'aménagement du territoire, un conseil d'administration rassemblant à nouveau des représentants des deux secteurs.

Sans le dire avec autant de mots, et parfois même en disant le contraire de ce que la formule contenait vraiment, les promoteurs de cette stratégie escomptaient effectivement la fin de l'expérience des MRC pour enfin retrouver une liberté de manœuvre et d'action qui ne soit pas constamment engluée dans des discussions interminables et dans des exercices de concertation qui consomment énergie sans porter fruits. Si la formule devait se généraliser à l'ensemble des MRC mixtes, le divorce serait pratiquement consommé. Ne resterait qu'une garde partagée clairsemée.

Le groupe de travail n'a pas retenu cette
dernière stratégie. Il a plutôt opté pour une
stratégie qui consiste à réaménager le cadre
actuel des MRC. Ce réaménagement consiste
essentiellement à proposer des mesures
techniques pour faciliter la coexistence des
«partenaires obligés » des MRC mixtes.


Le groupe de travail n'a pas retenu cette dernière stratégie. Il a plutôt opté pour une stratégie qui consiste à réaménager le cadre actuel des MRC. Ce réaménagement consiste essentiellement à proposer des mesures techniques pour faciliter la coexistence des «partenaires obligés » des MRC mixtes. Pour introduire le compte rendu de ces mesures, voici le diagnostic que pose le groupe de travail:

«Pour la moitié des MRC soumises à notre étude, cette évolution vers une plus grande implication des MRC s'est réalisée sans heurts sérieux ou conséquents. Pour un autre quart des MRC étudiées, les problèmes de fonctionnement ont été vécus et réglés à la satisfaction des membres sans que persistent des séquelles graves ou inguérissables. Enfin, pour le dernier quart de nos $28 \mathrm{MRC}$, les problèmes de fonctionnement ont été tels que le désir de mettre en commun des expériences, des efforts monétaires et une vision d'avenir a cédé la place à l'indifférence, à la suspicion, à l'opposition, voire même au conflit ouvert.

Le fait de ne pas vivre une tension insupportable ne signifie évidemment pas que ces MRC ne rencontrent aucun problème de fonctionnement. Tel n'est pas le cas...»

\section{Pourquoi un remède de cheval quand l'esthétique suffit?}

Les mandats confiés au groupe de travail Ruest-Jutras/ Nicolet par la ministre Harel ne laissent aucun doute quant à la nature et à l'ampleur des recommandations attendues. Ces mandats sont au nombre de quatre:

- Identifier les différents problèmes que pose l'organisation municipale actuelle et prévisible dans les MRC visées;

- Formuler, à l'égard de ces MRC, des recommandations relatives aux règles de prise de décision;

- Identifier, s'il y a lieu, les nouvelles responsabilités ou compétences que ces MRC pourraient prendre en charge ;

- Examiner le mode de financement de ces MRC et, entre autres, la possibilité d'attribuer aux MRC visées des sources de revenus autonomes telles que l'allocation d'une partie plus ou moins importante des montants découlant de la mesure de diversification des revenus municipaux prévue par le pacte fiscal.
Rien dans ces mandats qui incite à revoir de fond en comble le système municipal dans les 28 territoires sur lesquels évoluent les $28 \mathrm{MRC}$ et leurs partenaires socioéconomiques. Aussi, le groupe de travail ne sera pas plus catholique que le pape et cherchera, dans toute la mesure du possible, à centrer ses recommandations sur les dimensions techniques. Sauf pour ce qui est de sa sensibilité toute particulière pour les communautés dites rurales, sensibilité qui se manifeste dans une section du chapitre sur la mise en contexte et dans une des huit catégories de ses recommandations générales, le groupe de travail ne déborde pas de ses mandats.

Le mandat qui concerne l'examen du mode de financement des MRC est vite réglé. Les intervenants rencontrés sont fort réservés sur cette question. Il en va de même du groupe de travail qui opte pour le statu quo et plaide pour une flexibilité volontaire de la part des partenaires dans les formules de partage des coûts d'opération des MRC.

Le mandat sur les compétences est plus délicat. La question soulève des discussions animées et parfois passionnées dans un bon nombre de MRC. La problématique est simple, mais les solutions sont moins évidentes.

Partant de l'idée qu'un organisme vivant tend, tout naturellement, à vouloir progresser, il s'agit de se demander «si » et «comment » cet organisme, en l'occurrence la MRC, peut acquérir de nouvelles compétences, les mettre au service de l'ensemble des partenaires et les faire financer par l'ensemble des contribuables de façon juste et équitable. Au préalable, il faut évidemment avoir convenu que cet organisme mérite de vivre et de progresser. Sinon, la discussion devient rapidement futile ou bien les arguments invoqués sont des écrans qui occultent les vrais motifs.

Partant de l'idée qu'un organisme vivant tend, tout naturellement, à vouloir progresser, il s'agit de se demander «si » et «comment» cet organisme, en l'occurrence la MRC, peut acquérir de nouvelles compétences, les mettre au service de l'ensemble des partenaires et les faire financer par l'ensemble des contribuables de façon juste et équitable. Au préalable, il faut évidemment avoir convenu que cet organisme mérite de vivre et de progresser. Sinon, la discussion devient rapidement futile ou bien les arguments invoqués sont des écrans qui occultent les vrais motifs. 
Le groupe de travail ne se prononce pas sur le «si », c'est-à-dire sur les compétences supplémentaires qu'il convient, ou non, de confier aux $28 \mathrm{MRC}$, par législation ou par toute autre forme de délégation. Il recommande plutôt des mesures pour encadrer «comment» l'acquisition de nouvelles compétences devrait se faire et s'administrer. D'abord un principe: toute délégation de nouvelles compétences, à l'exception des compétences obligatoires que le gouvernement impose aux MRC par législation, doit être faite en faveur des municipalités locales qui sont alors libres d'en confier la gestion à la MRC ou de la gérer en propre. Ensuite des modalités: eu égard au droit de retrait des municipalités et eu égard à la participation de tous les partenaires au financement des compétences librement déléguées à la MRC.

Les mandats sur l'identification des problèmes d'organisation des MRC et sur le processus de prise de décision dans ces dernières sont deux mandats très chauds, sinon brûlants.

Comme nous avons tenté de le souligner plus haut, les difficultés à vivre ensemble trouvent une échappatoire au moment de prendre des décisions qui engagent, et les urbains et les ruraux, même sur des sujets relativement banals ou anodins. Les suspicions, fondées ou imaginées, des uns à l'égard des autres s'expriment alors librement et risquent de paralyser le travail des MRC. C'est pourquoi le groupe de travail n'a pas cherché à minimiser les problèmes de fonctionnement liés au processus de prise de décision et au mode de représentation des deux groupes de municipalités au sein des instances décisionnelles de la MRC et, par extension, au sein des CLD (Centres locaux de développement).

Toutefois, en conformité avec le traitement de ses autres mandats, le groupe de travail ne propose pas de modifications radicales au mode actuel de fonctionnement des MRC. Il recommande plutôt des mesures d'accommodement. Ainsi, la reconduction de la formule de la double majorité est assortie d'une proposition «mathématique » visant à faire en sorte que la ville centre, actuelle ou à venir au terme du regroupement de l'agglomération de recensement, ne puisse jamais détenir plus de voix (volet de la représentation politique) que l'ensemble des autres municipalités au sein du conseil des maires de la MRC. D'où l'obligation, pour la ville centre, de réaliser des alliances ou des compromis avec des intervenants ruraux pour être en mesure, non pas de bloquer, mais d'initier une action par l'intermédiaire de la MRC.
Même prudence pour ce qui est du mode d'organisation des MRC: reconduction du mode actuel d'élection du préfet accompagnée de deux recommandations visant à faire en sorte que les deux groupes de municipalités en présence puissent occuper ce poste en tandem ou en alternance; recommandation pour retrouver ce même équilibre au sein des comités administratifs et des autres comités à l'œuvre dans les MRC; enfin, recommandation en faveur d'habiliter les MRC qui voudraient avoir recours à la «formule Arthabaska» de pouvoir le faire en respectant certaines règles au cas où un blocage se manifesterait au cours du processus de décision à cet effet.

Au terme de ces recommandations générales, le groupe de travail a pris l'initiative de formuler des recommandations spécifiques pour toutes et chacune des $28 \mathrm{MRC}$ étudiées. Il s'agit essentiellement de transcrire, en les adaptant aux situations concrètes, l'une et l'autre des recommandations «prudentielles» qui sont la marque de commerce du groupe de travail Ruest-Jutras/Nicolet.

\section{Conclusion sur le sort réservé aux groupes de travail}

Un groupe de travail comme celui qui fait l'objet du présent texte est essentiellement constitué pour formuler des avis pratiques. Ses mandats ainsi que son échéancier sont dictés par les contraintes de la démarche législative et par la volonté politique du parti au pouvoir.

\section{Il est donc trop tôt pour évaluer combien des recommandations du groupe de travail auront été retenues à la fin du processus. II est tout de même permis de dire que le texte de l'avant-projet de loi comprenait plusieurs des recommandations du groupe de travail.}

Le groupe de travail coprésidé par madame Ruest-Jutras et par monsieur Nicolet ayant été institué pour donner des avis pratiques et techniques sur des sujets bien circonscrits en rapport avec la réforme municipale en cours, il importait que la remise de son rapport respecte les exigences de la démarche législative. Malgré la prolongation de son mandat, le groupe de travail déposa son rapport avant que la machine administrative et juridique ne rédige la dernière mouture des projets de loi devant être déposés à l'Assemblée nationale en décembre 2001. Il aurait toutefois pu être laissé sur le carreau, n'eut été de la volonté politique qui animait le gouvernement et la 
ministre des Affaires municipales et de la Métropole eu égard à la réforme municipale entreprise avec détermination depuis trois ou quatre ans.

Au moment de rédiger cette note (mi-mai), la Commission parlementaire chargée de l'étude du projet de loi 77, soit la Loi modifiant diverses dispositions législatives concernant les municipalités régionales de comté vient tout juste de terminer ses travaux. Il est donc trop tôt pour évaluer combien des recommandations du groupe de travail auront été retenues à la fin du processus. Il est tout de même permis de dire que le texte de l'avantprojet de loi comprenait plusieurs des recommandations du groupe de travail.

Sur le mode d'organisation des MRC, le texte de l'avantprojet de loi reformule la recommandation visant à faire en sorte que les deux groupes de municipalités en présence puissent occuper le poste de préfet en tandem ou en alternance, ainsi que celle visant à équilibrer la représentation au sein des comités administratifs et des autres comités à l'œuvre dans les MRC, en particulier dans le but de ne pas exclure la présence d'un représentant de la ville centre. Sur la formule d'encadrement du vote à la double majorité, le texte de l'avant-projet de loi s'apparente à celui que le groupe de travail avait proposé. Il en va de même pour les recommandations ayant pour objectif de dénouer des impasses quant à l'adoption des budgets d'opération et quant aux modalités et conditions administratives et financières relatives à l'acquisition et à la gestion de nouvelles compétences par les MRC mixtes.
Bref, sous réserve d'une analyse comparative plus fine des deux textes, une fois que le processus sera complètement terminé, il est permis de conclure que le groupe de travail n' aura pas travaillé en vain. A-t-il été plus performant que d'autres groupes de travail ou comités d'étude sur ce plan? Nous ne connaissons pas d'évaluations scientifiques sur ce thème. Est-ce que les conditions qui ont présidé à sa mise sur pied et à sa réalisation étaient exceptionnellement favorables ? Probablement. Enfin, est-ce que la démarche dans laquelle s'est inscrit le groupe de travail est celle qui devrait être la démarche normale lorsqu'il s'agit d'élaborer un nouvelle politique publique. En théorie, oui. En pratique, l'élaboration des politiques publiques obéit peu souvent à un processus linéaire du type de celui dont a bénéficié le groupe de travail RuestJutras/Nicolet: identification de problèmes, recherche organisée et ouverte de solutions à ces problèmes, mise à l'agenda législatif des recommandations formulées au terme de cette recherche, adoption de la nouvelle législation par l'Assemblée Nationale et mise en œuvre de cette législation.

Une fois n'est pas coutume! La réforme municipale contient tout, et un peu plus!

\section{Note}

1 Jacques Léveillée est professeur à l'Université du Québec à Montréal (sciences politiques) et chercheur associé au Groupe de recherche sur l'innovation municipale (GRIM). 